\title{
The Relationship of CSF and Plasma Cytokine Levels in HIV Infected Patients with Neurocognitive Impairment
}

\author{
Lin Yuan, ${ }^{1}$ An Liu, ${ }^{1}$ Luxin Qiao, ${ }^{2}$ Bo Sheng, ${ }^{1}$ Meng Xu, ${ }^{1}$ Wei Li, ${ }^{1}$ and Dexi Chen ${ }^{2}$ \\ ${ }^{1}$ Center for Infectious Disease, Beijing Youan Hospital, Capital Medical University, Beijing 100069, China \\ ${ }^{2}$ Beijing Institute of Liver Disease, Beijing 100069, China \\ Correspondence should be addressed to Wei Li; wei.advance@gmail.com and Dexi Chen; dexi0963@yahoo.com
}

Received 9 December 2014; Revised 10 February 2015; Accepted 15 February 2015

Academic Editor: Lucette A. Cysique

Copyright (c) 2015 Lin Yuan et al. This is an open access article distributed under the Creative Commons Attribution License, which permits unrestricted use, distribution, and reproduction in any medium, provided the original work is properly cited.

\begin{abstract}
Although HAD is now rare due to HAART, the milder forms of HAND persist in HIV-infected patients. HIV-induced systemic and localized inflammation is considered to be one of the mechanisms of HAND. The levels of cytokines in CSF were associated with neurocognitive impairment in HIV infection. However, the changes of cytokines involved in cognition impairment in plasma have not been shown, and their relationships between CSF and plasma require to be addressed. We compared cytokine levels in paired CSF and plasma samples from HIV-infected individuals with or without neurocognitive impairment. Cytokine concentrations were measured by Luminex xMAP. In comparing the expression levels of cytokines in plasma and CSF, IFN- $\alpha 2$, IL-8, IP-10, and MCP-1 were significantly higher in CSF. Eotaxin was significantly higher in plasma, whereas G-CSF showed no difference between plasma and CSF. G-CSF $(P=0.0079)$, IL-8 $(P=0.0223)$, IP-10 $(P=0.0109)$, and MCP-1 $(P=0.0497)$ in CSF showed significant difference between HIV-CI and HIV-NC group, which may indicate their relationship to HIV associated neurocognitive impairment. In addition, G-CSF $(P=0.0191)$ and IP-10 $(P=0.0377)$ in plasma were significantly higher in HIV-CI than HIV-NC. The consistent changes of G-CSF and IP-10 in paired plasma and CSF samples might enhance their potential for predicting HAND.
\end{abstract}

\section{Introduction}

HIV associated neurocognitive disorder (HAND) is a prevalent and significant challenge to HIV infected populations [1]. Its clinical severity includes asymptomatic neurocognitive impairment (ANI), mild neurocognitive disorder (MND), and more severe HIV associated dementia (HAD) [2]. The introduction of highly antiretroviral therapy (HAART) has effectively reduced the mortality and morbidity related to HIV; however, the overall prevalence of HAND remains high. More than $50 \%$ of HIV infected persons receiving HAART demonstrate milder HAND syndromes [1]. Progressive neurocognitive disorders in HIV patients are related with impaired quality of life [3-6], poorer antiretroviral adherence [7-11], and higher mortality [12]. The pathogenic mechanism of HAND has not been clear. Inflammatory response in the periphery and central nervous system (CNS) where monocyte/macrophage $(\mathrm{M} / \mathrm{M})$ is activated has been correlated with HAND $[13,14]$. Inflammatory factors and chemokines expressed by activated $\mathrm{M} / \mathrm{M}$ play a critical role in neuroinflammation and neurodegeneration in HAND [1517]. In our previous study we demonstrated the elevation of a small panel of cytokines in CSF was correlated with neurocognitive impairment in HIV infected patients [18]. However, there is still lack of data addressing the relationship between blood and CSF concentrations of cytokines in relation to HAND.

Thus, this study aimed to firstly determine whether CSF levels of the inflammatory cytokine or chemokine were elevated in HIV infected patients with neurocognitive impairment and secondly to examine relationships between the concentrations of these cytokines in the CSF and plasma.

\section{Methods}

2.1. Study Participants. HIV infected patients were enrolled in Henan and Yunnan provinces in China, and CSF were acquired at the time of a clinically indicated lumbar puncture. CSF and plasma samples were taken simultaneously. All the samples were centrifuged, and cell-free CSF and plasma were 
TABLE 1: Demographic and disease characteristics of subjects.

\begin{tabular}{|c|c|c|c|}
\hline Characteristics & HIV-CI & HIV-NC & $P$ value \\
\hline Number of subjects & 52 & 33 & \\
\hline Age in years [median (IQR)] & $38(31-42)$ & $38(33-42)$ & \\
\hline \multicolumn{4}{|l|}{ Gender $[n(\%)]$} \\
\hline Male & $38(73 \%)$ & $21(64 \%)$ & \\
\hline Female & $14(27 \%)$ & $12(36 \%)$ & \\
\hline \multicolumn{4}{|l|}{ HIV RNA (log 10 copies/mL) [median (IQR)] } \\
\hline CSF & $4.50(2.95-7.50)$ & $3.06(1.70-3.60)$ & $P=0.3640$ \\
\hline Plasma & $5.915(3.44-7.50)$ & $3.905(2.70-5.21)$ & $P=0.8194$ \\
\hline CD4+ cell count $($ cell $/ \mu \mathrm{L})[$ median (IQR)] & $41(21-120)$ & $69(21-120)$ & $P=0.3275$ \\
\hline \multicolumn{4}{|l|}{ CSF } \\
\hline Cell count $($ cell $/ \mu \mathrm{L})[$ median (IQR)] & $12(6-21)$ & $11(7-30)$ & $P=0.7499$ \\
\hline Protein $(\mathrm{mg} / \mathrm{mL})$ [median (IQR)] & $74(73-110)$ & $41(34-48)$ & $P=0.0097$ \\
\hline Number of threated by HAART $[n(\%)]$ & $20(38.5)$ & $17(51.5)$ & \\
\hline Treatment duration (months) [median (range)] & $4(2-6)$ & $6(2-18)$ & \\
\hline CNS complications $[n(\%)]$ & $17(33 \%)$ & $12(36 \%)$ & \\
\hline
\end{tabular}

aliquot and immediately frozen at $-80^{\circ} \mathrm{C}$. The Memorial Sloan-Kettering Scale (MSK) was used to categorize the neurocognitive impairment of each patient. This study was approved by ethics committees at the Capital University of Medical Science in Beijing, and all participants provided informed consent.

2.2. Measurement of CSF Cytokines. A total of 6 cytokines were measured in paired CSF and plasma samples using human cytokine/chemokine MILLIplex kits (Millipore Corp, Billerica, MA, USA): IL-8, eotaxin, granulocyte colonystimulating factor (G-CSF), interferon- (IFN-) $\alpha 2$, IFN$\gamma$-induced protein- (IP-) 10 , and monocyte chemotactic protein- (MCP-) 1. The lower levels of detection were ranged between 0.2 and $10.1 \mathrm{pg} / \mathrm{mL}$ for each of the cytokines measured. All samples were assayed concurrently in duplicate. Data were collected using a Bio-Plex Suspension Array Reader (Bio-Rad Laboratories Inc., Hercules, California, USA). Cytokine concentrations that were lower than the lower limits of detection were reported as undetectable (0).

2.3. Statistical Analyses. Univariate analyses were performed using GraphPad Prism 5 (GraphPad Software, San Diego, California, USA). Mann-Whitney $U$ test and Wilcoxon signed rank test were used for unmatched and matched comparisons, respectively. Spearman rank tests were used to test for correlations. $P$ values less than 0.05 were considered significant.

\section{Results}

There were $85 \mathrm{HIV}-1$ clade $\mathrm{B}$ or $\mathrm{B}^{\prime}$ infected patients enrolled with paired CSF and plasma samples collected for cytokine analysis. The median age was 38 years (range, $11-76$ years) and $59(69 \%)$ were men. The risk factors for HIV infection were sexual transmission $(n=44)$, intravenous drug use $(n=15)$, blood transfusion $(n=7)$, paid blood or plasma donation $(n=2)$, mother-to-child transmission $(n=2)$, and unknown $(n=15)$. In total, 29 of 85 patients had complications, including cryptococcal meningitis $(n=21)$, tuberculous meningitis $(n=2)$, Toxoplasma encephalitis $(n=2)$, Pneumocystis pneumonia $(n=2)$, and cytomegalovirus radiculitis $(n=2)$. Nearly half of the HIV infected patients (43\%) were receiving HAART at the time of evaluation. All were on multidrug combination ART regimens, which consisted of at least 2 NRTIs (e.g., AZT, D4T, 3TC, DDI, and TDF) plus an NNRTI (NVP or EFV) or a PI (LPV). None were on mono- or dualtherapy. Based on MSK classification, patients were classified into HIV infected with normal cognition (HIV-NC; $n=33$, MSK $=0$ ) and impaired cognition groups (HIV-CI; $n=52$ ). The HIV-CI group consisted of MSK $=0.5(n=24)$, MSK $=1(n=12)$, MSK $=2(n=10)$, and MSK = $3(n=6)$. The viral burden in CSF or plasma of HIV-CI group was higher than that of HIV-NC group, accompanied with lower CD 4 cell count. However, there was no statistically significant difference whether in viral load or in CD4 cell count for two groups (Table 1).

3.1. Plasma and CSF Cytokines. HIV infected patients with neurocognitive impairment had elevated levels of cytokines in CSF and plasma in comparison to those with normal neurocognition (Table 1). There was significant difference in CSF levels of G-CSF $(P=0.0079)$, IL-8 $(P=0.0223)$, IP$10(P=0.0109)$, and MCP-1 $(P=0.0497)$ between HIV-CI and HIV-NC group (Figure 1(a)). However, in plasma only GCSF $(P=0.0191)$ and IP-10 $(P=0.0377)$ showed significant difference (Figure 1(b)). The concentrations of eotaxin and IFN- $\alpha 2$ did not show any significant difference between these two groups either in CSF or in plasma (data not shown).

3.2. Relationships between Cytokine Concentrations in CSF and Plasma. In HIV infected patients, the expression levels of IFN- $\alpha 2$, IL-8, IP-10, and MCP-1 in CSF were significantly higher than in plasma. In contrast, eotaxin was significantly higher in plasma. But there was no difference for G-CSF. The expression levels of several cytokines showed significant 

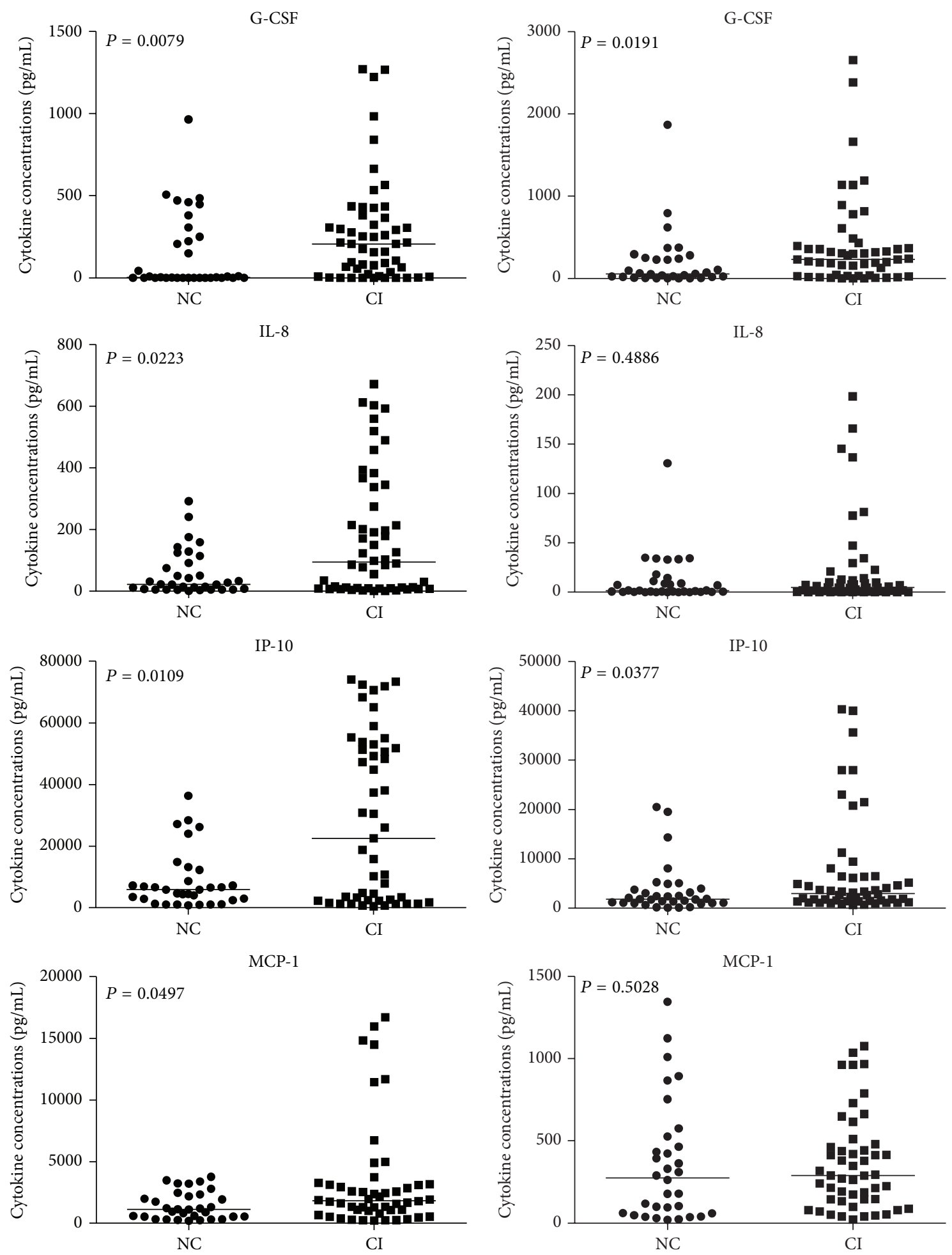

(a)

(b)

FIGURE 1: Comparison of G-CSF, IL-8, IP-10, and MCP-1 concentrations in paired CSF and plasma samples from 33 HIV infected subjects with normal cognition (HIV-NC) and 52 HIV infected subjects with cognition impairment (HIV-CI). Values below the lower limit of detection of the assay are reported as zero. The levels of G-CSF, IL-8, IP-10, and MCP-1 in CSF from HIV-CI group were significantly higher than HIV-NC group (a), whereas in plasma samples, only G-CSF and IP-10 showed significant difference (b). Differences were analyzed by the Mann-Whitney $U$ test. $P$ values of $<0.05$ were considered significant. Dots, cytokine level in CSF for each study subject; horizontal lines, median values for each group. 
TABLE 2: Cytokine concentrations in paired CSF and plasma samples.

\begin{tabular}{|c|c|c|c|c|c|c|c|c|}
\hline & \multicolumn{4}{|c|}{ HIV-CI } & \multicolumn{4}{|c|}{ HIV-NC } \\
\hline & Plasma & CSF & $\begin{array}{c}\text { Wilcoxon } \\
\text { signed rank, } \\
P\end{array}$ & $\begin{array}{l}\text { Correlation } \\
\text { coefficient }^{*}\end{array}$ & Plasma & CSF & $\begin{array}{c}\text { Wilcoxon } \\
\text { signed rank, } \\
P\end{array}$ & $\begin{array}{l}\text { Correlation } \\
\text { coefficient }^{*}\end{array}$ \\
\hline \multicolumn{9}{|l|}{ Eotaxin } \\
\hline Median & 78.86 & 0.94 & $<0.0001$ & -0.3949 & 59.36 & 0.79 & 0.0019 & -0.6241 \\
\hline IQR & $15.88-202.3$ & $0-45.9$ & & $P=0.0038$ & $6.54-200.4$ & $0-1.165$ & & $P=0.0001$ \\
\hline$<$ Detection Limit & $2 \%$ & $56 \%$ & & & $0 \%$ & $37 \%$ & & \\
\hline \multicolumn{9}{|l|}{ G-CSF } \\
\hline Median & 234.8 & 207 & 0.4255 & 0.1667 & 64.3 & 4.36 & 0.2436 & 0.5353 \\
\hline IQR & $31.54-421.5$ & $26.53-415.2$ & & $P=0.2375$ & $17.62-287.5$ & $0-279.2$ & & $P=0.0013$ \\
\hline$<$ Detection Limit & $4 \%$ & $13 \%$ & & & $8 \%$ & $27 \%$ & & \\
\hline \multicolumn{9}{|l|}{ IFN- $\alpha 2$} \\
\hline Median & 29.03 & 66.79 & 0.0039 & 0.6903 & 10.82 & 40.58 & 0.0174 & 0.4831 \\
\hline IQR & $5.59-80.2$ & $0.74-175.7$ & & $P<0.0001$ & $0-55.05$ & $0-104.6$ & & $P=0.0044$ \\
\hline$<$ Detection Limit & $10 \%$ & $25 \%$ & & & $23 \%$ & $17 \%$ & & \\
\hline \multicolumn{9}{|l|}{ IL-8 } \\
\hline Median & 4.78 & 100.6 & $<0.0001$ & -0.07862 & 1.47 & 22.44 & $<0.0001$ & 0.1896 \\
\hline IQR & $0.71-13.82$ & $8.86-343.4$ & & $P=0.5796$ & $0.375-12.82$ & $5.92-102.4$ & & $P=0.2907$ \\
\hline$<$ Detection Limit & $29 \%$ & $4 \%$ & & & $29 \%$ & $2 \%$ & & \\
\hline \multicolumn{9}{|l|}{ IP-10 } \\
\hline Median & 2985 & 17260 & $<0.0001$ & 0.7525 & 1845 & 5834 & 0.001 & 0.1756 \\
\hline IQR & $1451-6428$ & $1828-51620$ & & $P<0.0001$ & 1046-3891 & $2521-11330$ & & $P=0.3364$ \\
\hline$<$ Detection Limit & $0 \%$ & $6 \%$ & & & $0 \%$ & $2 \%$ & & \\
\hline \multicolumn{9}{|l|}{ MCP-1 } \\
\hline Median & 292.1 & 1755 & $<0.0001$ & 0.1527 & 289.4 & 1120 & $<0.0001$ & 0.08761 \\
\hline IQR & $144-502.9$ & $692.9-3147$ & & $P=0.2798$ & $60.25-550.8$ & $526.9-2294$ & & $P=0.6335$ \\
\hline$<$ Detection Limit & $0 \%$ & $4 \%$ & & & $0 \%$ & $2 \%$ & & \\
\hline
\end{tabular}

* Spearman rank correlation, values below the lower limits of detection set at zero.

correlations between CSF and plasma. Among them IP-10 presented the strongest correlation in the patients with HIVCI (correlation coefficient $0.7525, P<0.0001$ ) (Table 2).

\section{Discussion}

We demonstrated that the concentrations of G-CSF, IL8, IP-10, and MCP-1 in CSF samples from neurocognitive impaired HIV-1 infected patient were significantly higher than patients with normal cognition. This confirmed our finding in previously published study [18]. Elevated concentrations of cytokines that we examined reflected the activation of the immune response in central nervous system or periphery, which might provide a cytokine panel for predicting neurocognitive impairment in HIV-1 infected individuals. Clinical studies on the role of inflammation in HIV1 associated neurocognitive disorder rely on determinations of biomarkers in clinically accessible fluid compartments. CSF sample is not more likely to reach as plasma sample. In this study, there was not any correlation between paired CSF and plasma concentrations for any of the cytokines involved. This might indicate a limitation of plasma samples in reflecting the immune response in CNS. Fortunately, we found the concentrations of G-CSF and IP-10 in plasma of HIV-CI patient were higher than HIV-NC patients. The advantages of plasma sample, such as being most accessible and being able to be measured repeatedly, will enhance the potential of plasma G-CSF and IP-10 for predicting HIV1 related cognitive impairment. Additionally, the site of the highest cytokine level for each cytokine was various in plasma or CSF. IL-8, IFN- $\alpha 2$, IP-10, and MCP-1 were higher in CSF than plasma samples, whereas eotaxin and G-CSF were significantly higher in plasma. Taking into account the lack of correlation between CSF and plasma cytokine concentrations, these might suggest that some cytokines may be localized within the CSF which is the potential origin of immune response rather than resulting from the dissemination of cytokines via blood stream.

Interferon- $\gamma$-inducible protein 10, IP-10 (in the systematic nomenclature, CXCL10), is expressed by astrocytes, microglia, and endothelial cells in an inflammatory lesion and cells culture supernatants, acts specifically on activated $\mathrm{T}$ cells and macrophages, and attracts $\mathrm{T}$ lymphocytes into the CSF. Expression of IP-10 in the brain is correlated closely with increased infiltrating cells in herpes simplex virus (HSV), MHV, and West Nile virus (WNV) infection [19-21]. 
Abrogation of IP-10 expression by either depletion with antiIP-10 or genetic silencing dramatically reduces infiltration of T cell into the CNS. In the brain, IP-10 can be induced by HIV1 viral gp120, Nef, and Tat [22-24]. Cooperative interaction of HIV Tat and IFN- $\gamma$ results in IP-10 overexpression, which in turn can amplify the inflammatory responses within the CNS of HAD patients by recruiting more lymphocytes into the brain. IP-10 has been detected in the CSF of individuals with HIV-1 infection [25] and in the astrocytes in brains of individuals with HIV associated dementia [26]. It was also associated with SHIV encephalitis in macaques [27]. Upregulation of IP-10 may contribute to the BBB permeability changes and instruction of innate immune responses in CNS [28]. Zhang et al. indicated that IP-10 could intensify injury to the blood-brain barrier by NK cells via its receptor CXCR3 [29]. Exogenous CXCL10 led to increased membrane permeability in neurons and caused cell loss in the fetal brain cultures, which might be responsible for induction of neuron apoptosis [30]. In our study, we demonstrated the expression of IP-10 was significantly elevated in HIV-1 infected patients with neurocognitive impairment whether in CSF or in plasma. In addition, the expression levels of IP-10 in CSF and plasma in the patients with HIV-CI were significantly correlated (correlation coefficient $0.7525, P<$ 0.0001 ), but there was no correlation in HIV-NC patients. It suggested that neurocognitive impairment in HIV-1 infected patients might be accompanied by the arising of bloodbrain barrier (BBB) impairment. Increased permeability of $\mathrm{BBB}$ results in infiltration of activated $\mathrm{T}$ lymphocyte from periphery and overexpression of cytokines in CNS, which might promote the degeneration of neuron. Further studies on relationship between IP-10 and BBB injury are required.

There are some limitations to our study. Firstly, cases with HIV complication have not been excluded from the enrolled subjects (29/85), which might raise the possibility that an increase in cytokine levels with complications could explain their higher values. However, the ratios of cases with complication were almost equal in HIV-CI and HIVNC groups, with $33 \%$ and 36\%, respectively, making this explanation unlikely. Secondly, the patients in this study have relative lower CD4 T cell count [53 (20-120), median (IQR)]. There was no significant difference in $\mathrm{CD} 4$ cell count between HIV-CI and HIV-NC patients; nevertheless, relative poorer immune state could exert a negative influence on inflammatory response.

In conclusion, our study suggested that elevated levels of G-CSF, IL-8, IP-10, and MCP-1 in CSF may indicate HIV associated neurocognitive impairment. Consistent changes of G-CSF and IP-10 in paired plasma and CSF samples enhance their potential for predicting HAND.

\section{Conflict of Interests}

The authors declare that there is no conflict of interests regarding the publication of this paper.

\section{Authors' Contribution}

Lin Yuan and An Liu contributed equally to this work.

\section{Acknowledgments}

This work was supported by grants from Beijing Municipal Science and Technology Program (Z141100002114037, Z131107002213071), Chinese National Twelfth Five Year Key Scientific and Technological Project (2012ZX10001-002, 2012ZX10001-003, 2012ZX10001-004, and 2012BAI15B08), National Natural Science Foundation of China (81371399, 81371332, and 81200848), Beijing Natural Science Foundation (7142078), and AIDS Research Beijing Key Laboratory (BZ0089).

\section{References}

[1] R. K. Heaton, D. B. Clifford, D. R. Franklin et al., "HIVassociated neurocognitive disorders persist in the era of potent antiretroviral therapy: charter study," Neurology, vol. 75, no. 23, pp. 2087-2096, 2010.

[2] A. Antinori, G. Arendt, J. T. Becker et al., "Updated research nosology for HIV-associated neurocognitive disorders," Neurology, vol. 69, no. 18, pp. 1789-1799, 2007.

[3] S. Evans, S. Ferrando, M. Sewell, K. Goggin, B. Fishman, and J. Rabkin, "Pain and depression in HIV illness," Psychosomatics, vol. 39, no. 6, pp. 528-535, 1998.

[4] W. C. Holmes, B. Bix, M. Meritz, J. Turner, and C. Hutelmyer, "Human immunodeficiency virus (HIV) infection and quality of life: the potential impact of Axis I psychiatric disorders in a sample of 95 HIV seropositive men," Psychosomatic Medicine, vol. 59, no. 2, pp. 187-192, 1997.

[5] C. D. Sherbourne, R. D. Hays, J. A. Fleishman et al., "Impact of psychiatric conditions on health-related quality of life in persons with HIV infection," The American Journal of Psychiatry, vol. 157, no. 2, pp. 248-254, 2000.

[6] J. D. Piette, J. A. Fleishman, M. D. Stein, V. Mor, and K. Mayer, "Perceived needs and unmet needs for formal services among people with HIV disease," Journal of Community Health, vol. 18, no. 1, pp. 11-23, 1993.

[7] B. Spire, S. Duran, M. Souville, C. Leport, F. Raffi, and J.P. Moatti, "Adherence to highly active antiretroviral therapies (HAART) in HIV-infected patients: From a predictive to a dynamic approach," Social Science and Medicine, vol. 54, no. 10, pp. 1481-1496, 2002.

[8] J. Bartlett, "Addressing the challenges of adherence," Journal of Acquired Immune Deficiency Syndromes, vol. 29, supplement 1, pp. S2-S10, 2002.

[9] M. A. Chesney, J. Ickovics, F. M. Hecht, G. Sikipa, and J. Rabkin, "Adherence: a necessity for successful HIV combination therapy," AIDS, vol. 13, supplement, pp. S271-S278, 1999.

[10] V. Gordillo, J. Del Amo, V. Soriano, and J. González-Lahoz, "Sociodemographic and psychological variables influencing adherence to antiretroviral therapy," AIDS, vol. 13, no. 13, pp. 1763-1769, 1999.

[11] N. Singh, S. M. Berman, S. Swindells et al., "Adherence of human immunodeficiency virus-infected patients to antiretroviral therapy," Clinical Infectious Diseases, vol. 29, no. 4, pp. 824830, 1999.

[12] T. J. Mayne, E. Vittinghoff, M. A. Chesney, D. C. Barrett, and T. J. Coates, "Depressive affect and survival among gay and bisexual men infected with HIV," Archives of Internal Medicine, vol. 156, no. 19, pp. 2233-2238, 1996. 
[13] J. D. Glass, H. Fedor, S. L. Wesselingh, and J. C. McArthur, "Immunocytochemical quantitation of human immunodeficiency virus in the brain: correlations with dementia," Annals of Neurology, vol. 38, no. 5, pp. 755-762, 1995.

[14] H. Adle-Biassette, F. Chrétien, L. Wingertsmann et al., "Neuronal apoptosis does not correlate with dementia in HIV infection but is related to microglial activation and axonal damage," Neuropathology and Applied Neurobiology, vol. 25, no. 2, pp. 123-133, 1999.

[15] V. V. R. Bandaru, J. C. McArthur, N. Sacktor et al., "Associative and predictive biomarkers of dementia in HIV-1-infected patients," Neurology, vol. 68, no. 18, pp. 1481-1487, 2007.

[16] L. M. Bebell, J.-A. Passmore, C. Williamson et al., "Relationship between levels of inflammatory cytokines in the genital tract and $\mathrm{CD}^{+}$cell counts in women with acute HIV-1 infection," Journal of Infectious Diseases, vol. 198, no. 5, pp. 710-714, 2008.

[17] D. B. Clifford, A. M. Fagan, D. M. Holtzman et al., "CSF biomarkers of Alzheimer disease in HIV-associated neurologic disease," Neurology, vol. 73, no. 23, pp. 1982-1987, 2009.

[18] L. Yuan, L. Qiao, F. Wei et al., "Cytokines in CSF correlate with HIV-associated neurocognitive disorders in the post-HAART era in China," Journal of NeuroVirology, vol. 19, no. 2, pp. 144149, 2013.

[19] S. Molesworth-Kenyon, A. Mates, R. Yin, R. Strieter, J. Oakes, and R. Lausch, "CXCR3, IP-10, and Mig are required for CD4 $4^{+}$ T cell recruitment during the DTH response to HSV-1 yet are independent of the mechanism for viral clearance," Virology, vol. 333, no. 1, pp. 1-9, 2005.

[20] L. N. Stiles, M. T. Liu, J. C. Kane, and T. E. Lane, "CXCL10 and trafficking of virus-specific T cells during coronavirus-induced demyelination," Autoimmunity, vol. 42, no. 6, pp. 484-491, 2009.

[21] B. Zhang, K. C. Ying, B. Lu, M. S. Diamond, and R. S. Klein, "CXCR3 mediates region-specific antiviral $\mathrm{T}$ cell trafficking within the central nervous system during west nile virus encephalitis," Journal of Immunology, vol. 180, no. 4, pp. 26412649, 2008.

[22] G. van Marle, S. Henry, T. Todoruk et al., "Human immunodeficiency virus type $1 \mathrm{Nef}$ protein mediates neural cell death: a neurotoxic role for IP-10," Virology, vol. 329, no. 2, pp. 302-318, 2004.

[23] V. C. Asensio, J. Maier, R. Milner et al., "Interferonindependent, human immunodeficiency virus type 1 gp120mediated induction of CXCL10/IP-10 gene expression by astrocytes in vivo and in vitro," Journal of Virology, vol. 75, no. 15, pp. 7067-7077, 2001.

[24] O. Kutsch, J.-W. Oh, A. Nath, and E. N. Benveniste, "Induction of the chemokines interleukin- 8 and IP-10 by human immunodeficiency virus type 1 Tat in astrocytes," Journal of Virology, vol. 74, no. 19, pp. 9214-9221, 2000.

[25] V. J. Sanders, C. A. Pittman, M. G. White, G. Wang, C. A. Wiley, and C. L. Achim, "Chemokines and receptors in HIV encephalitis," AIDS, vol. 12, no. 9, pp. 1021-1026, 1998.

[26] H.-S. Suh, M. Cosenza-Nashat, N. Choi et al., "Insulin-like growth factor 2 receptor is an IFN $\gamma$-inducible microglial protein that facilitates intracellular HIV replication: implications for HIV-induced neurocognitive disorders," American Journal of Pathology, vol. 177, no. 5, pp. 2446-2458, 2010.

[27] Y. Sui, L. Stehno-Bittel, S. Li et al., "CXCL10-induced cell death in neurons: role of calcium dysregulation," European Journal of Neuroscience, vol. 23, no. 4, pp. 957-964, 2006.

[28] Y. Kuang, S. N. Lackay, L. Zhao, and Z. F. Fu, "Role of chemokines in the enhancement of $\mathrm{BBB}$ permeability and inflammatory infiltration after rabies virus infection," Virus Research, vol. 144, no. 1-2, pp. 18-26, 2009.

[29] Y. Zhang, Z. Gao, D. Wang et al., "Accumulation of natural killer cells in ischemic brain tissues and the chemotactic effect of IP10," Journal of Neuroinflammation, vol. 11, article 79, 2014.

[30] Y. Sui, R. Potula, N. Dhillon et al., "Neuronal apoptosis is mediated by CXCL10 overexpression in simian human immunodeficiency virus encephalitis," The American Journal of Pathology, vol. 164, no. 5, pp. 1557-1566, 2004. 

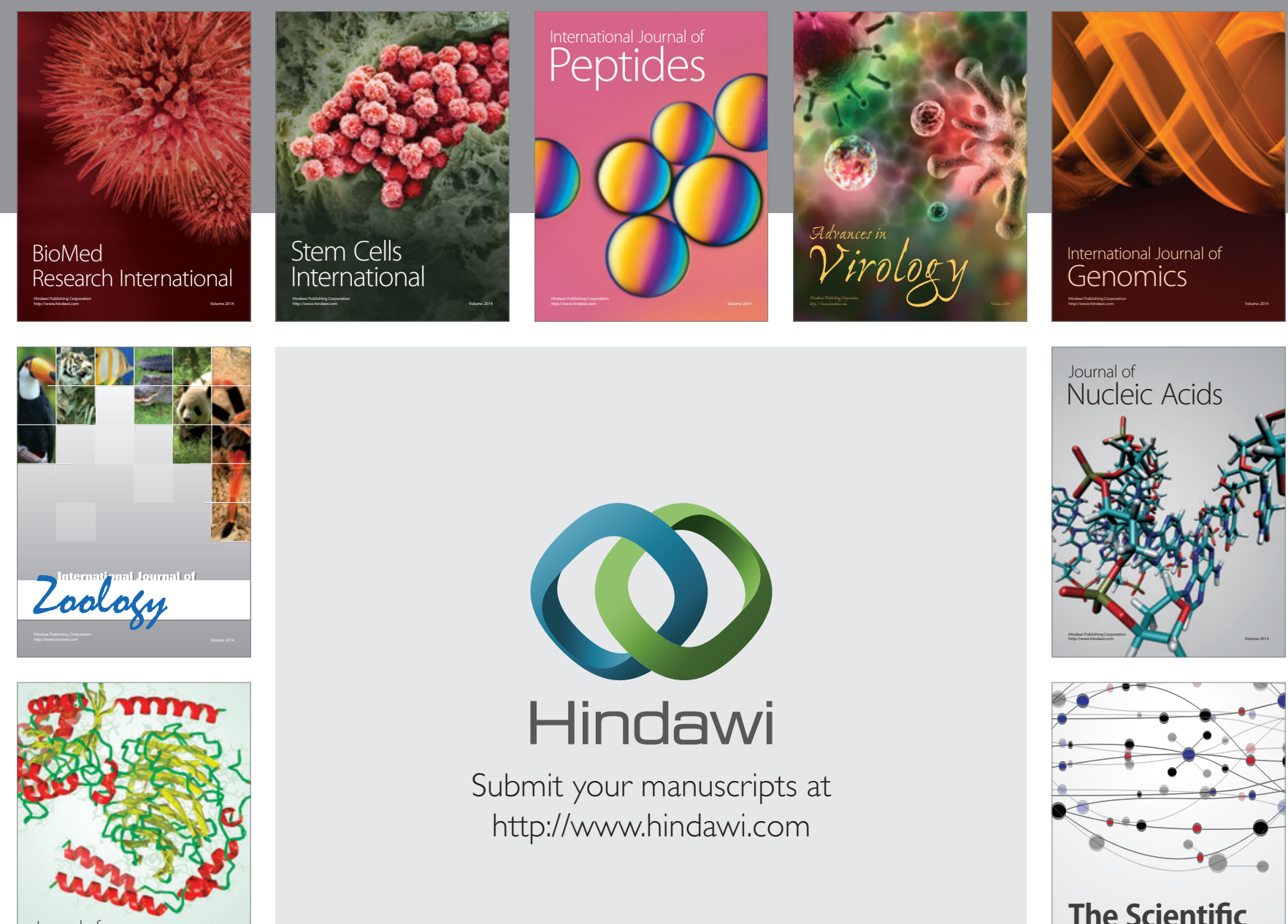

Submit your manuscripts at

http://www.hindawi.com

Journal of
Signal Transduction
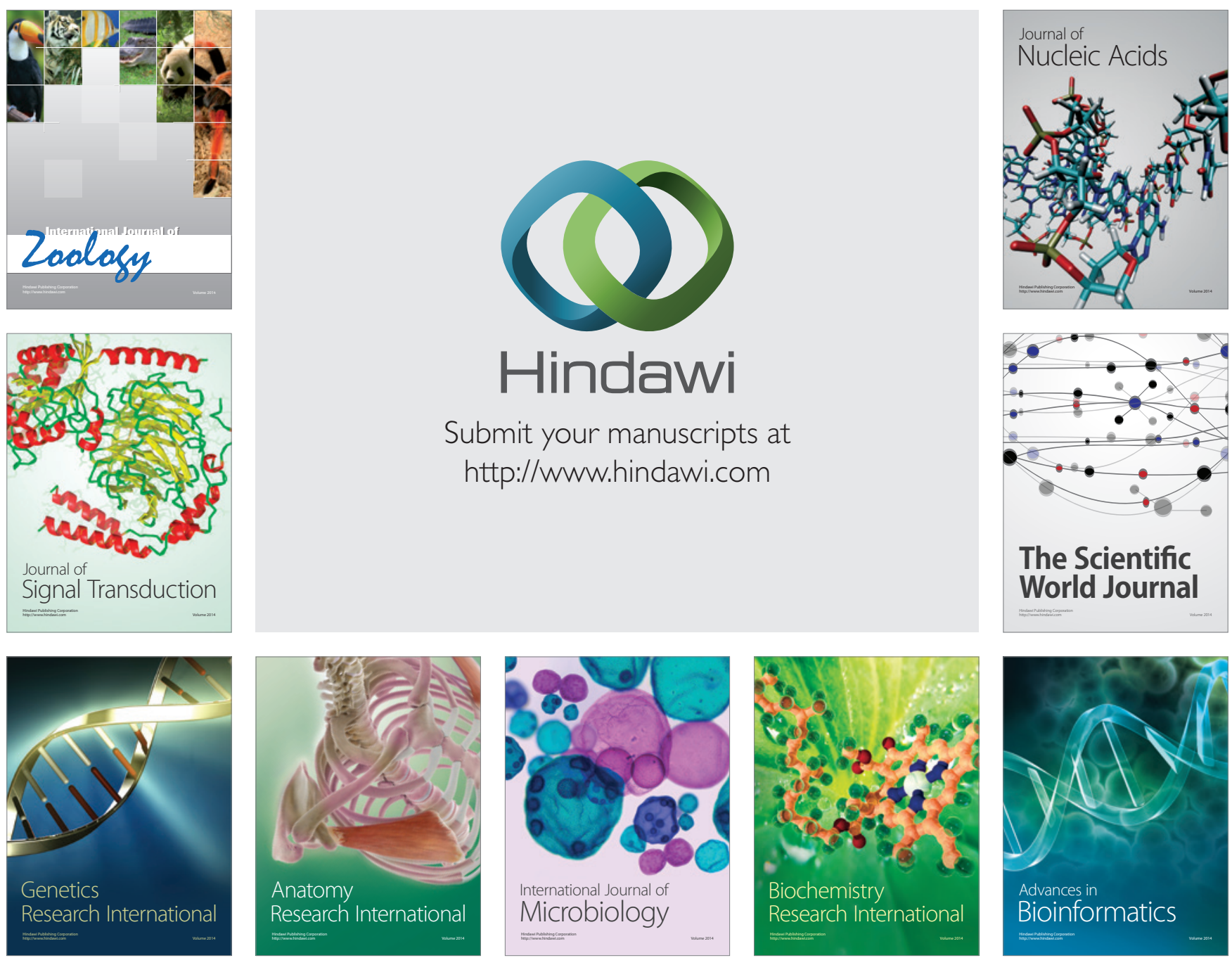

The Scientific World Journal
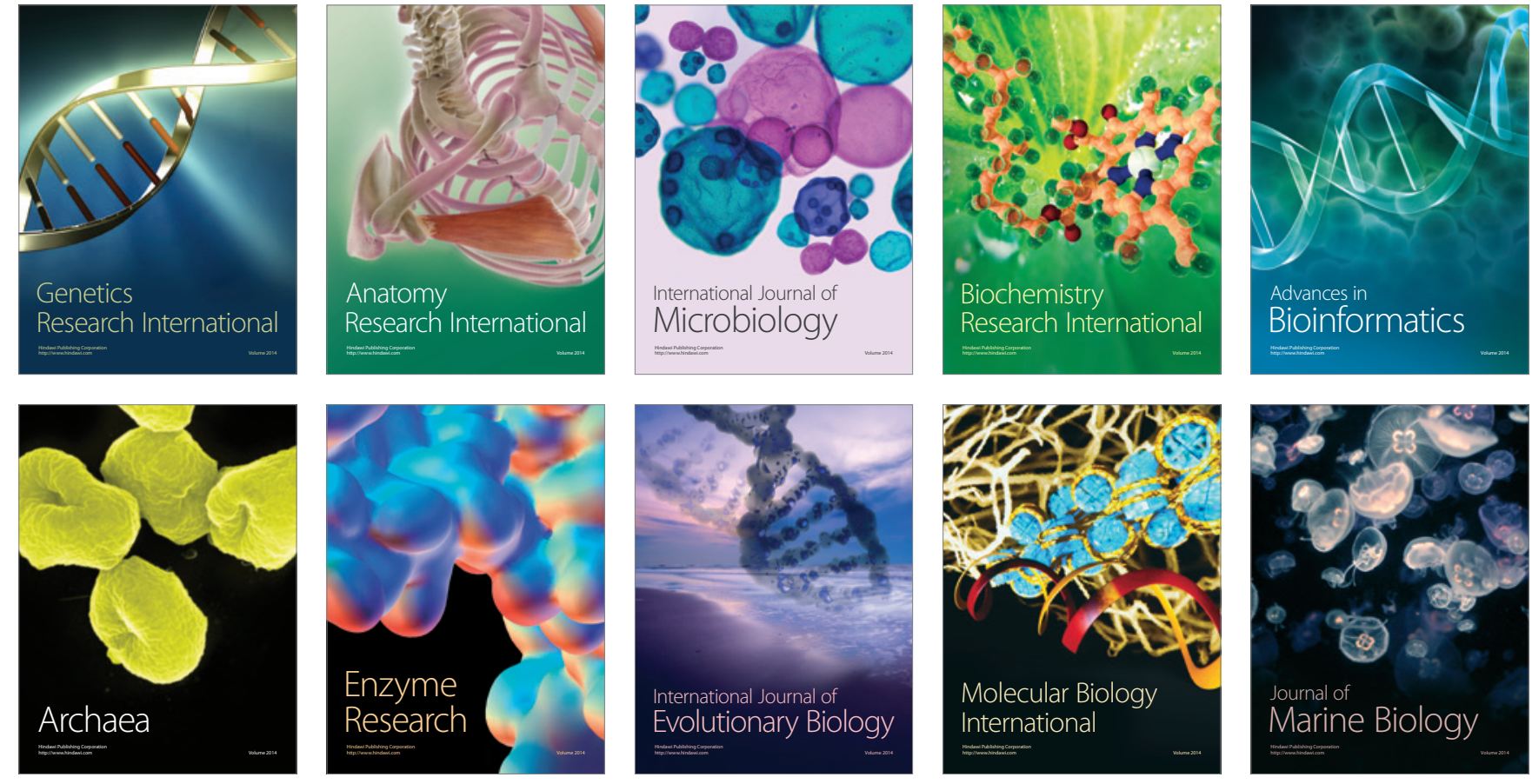